\title{
Some new Hardy-type inequalities on time scales
}

\author{
Ahmed A. El-Deeb ${ }^{1 *}$, Hamza A. Elsennary and Dumitru Baleanu ${ }^{2,3,4}$
}

"Correspondence:

ahmedeldeeb@azhar.edu.eg

'Department of Mathematics,

Faculty of Science, Al-Azhar

University, Nasr City, Cairo, 11884,

Egypt

Full list of author information is

available at the end of the article

\begin{abstract}
In this paper, we will prove some new dynamic inequalities of Hardy-type on time scales. Some of the integral and difference inequalities that will be derived from our results in the continuous and discrete cases are original. The main results will be proved by using the dynamic Hölder inequality, integration by parts formula on time scales, and Keller's chain rule on time scales. We will apply the main results to the continuous calculus, discrete calculus, and $q$-calculus as special cases.
\end{abstract}

MSC: 26D10; 26D15; 34N05; 26E70

Keywords: Hardy's inequality; Dynamic inequality; Time scale

\section{Introduction}

In 1920, Hardy [13] established the following renowned discrete inequality.

Theorem 1.1 If $\{a(n)\}_{n=0}^{\infty}$ is a nonnegative real sequence and $p>1$, then

$$
\sum_{n=1}^{\infty}\left(\frac{1}{n} \sum_{m=1}^{n} a(m)\right)^{p} \leq\left(\frac{p}{p-1}\right)^{p} \sum_{n=1}^{\infty} a^{p}(n) .
$$

Hardy discovered this inequality while attempting to sketch an easier proof of Hilbert's inequality for double series which was known at that time.

In 1925, using the calculus of variations, Hardy himself in [7] gave the integral analogue of inequality (1.1) as follows:

Theorem 1.2 Iff is a nonnegative continuous function on $[0, \infty)$ and $p>1$, then

$$
\int_{0}^{\infty}\left(\frac{1}{x} \int_{0}^{x} f(s) d s\right)^{p} d x \leq\left(\frac{p}{p-1}\right)^{p} \int_{0}^{\infty} f^{p}(x) d x
$$

In 1927, Littlewood and Hardy [25] extended inequality (1.1) and obtained the following discrete inequalities.

Theorem 1.3 Let $\{a(n)\}_{n=0}^{\infty}$ be a sequence of nonnegative real numbers.

(c) The Author(s) 2020. This article is licensed under a Creative Commons Attribution 4.0 International License, which permits use sharing, adaptation, distribution and reproduction in any medium or format, as long as you give appropriate credit to the original author(s) and the source, provide a link to the Creative Commons licence, and indicate if changes were made. The images or other third party material in this article are included in the article's Creative Commons licence, unless indicated otherwise in a credit line to the material. If material is not included in the article's Creative Commons licence and your intended use is not permitted by statutory regulation or exceeds the permitted use, you will need to obtain permission directly from the copyright holder. To view a copy of this licence, visit http://creativecommons.org/licenses/by/4.0/. 
(i) If $p>1$ and $\alpha>1$, then

$$
\sum_{n=1}^{\infty} \frac{1}{n^{\alpha}}\left(\sum_{m=1}^{n} a(m)\right)^{p} \leq C(p, \alpha) \sum_{n=1}^{\infty} \frac{1}{n^{\alpha-p}} a^{p}(n) .
$$

(ii) If $p>1$ and $\alpha<1$, then

$$
\sum_{n=1}^{\infty} \frac{1}{n^{\alpha}}\left(\sum_{m=n}^{\infty} a(m)\right)^{p} \leq C(p, \alpha) \sum_{n=1}^{\infty} \frac{1}{n^{\alpha-p}} a^{p}(n)
$$

where $C(p, \alpha)$ in inequalities (1.3) and (1.4) is a nonnegative constant that depends on $p$ and $\alpha$.

In the same paper [25], the authors proved the following sharp inequality of Hardy-type.

Theorem 1.4 Iff is a nonnegative continuous function on $[0, \infty)$ and $p>1$, then

$$
\int_{0}^{\infty}\left(\frac{1}{x} \int_{x}^{\infty} f(s) d s\right)^{p} d x \leq p^{p} \int_{0}^{\infty} f^{p}(x) d x
$$

which by a trivial transformation can be rewritten as

$$
\int_{0}^{\infty}\left(\int_{x}^{\infty} f(s) d s\right)^{p} d x \leq p^{p} \int_{0}^{\infty} x^{p} f^{p}(x) d x
$$

In 1928, Hardy [15] established the continuous versions of inequalities (1.3) and (1.4) as follows:

Theorem 1.5 Let $f$ be a nonnegative continuous function on $[0, \infty)$.

(i) If $p>1$ and $\alpha>1$, then

$$
\int_{0}^{\infty} \frac{1}{x^{\alpha}}\left(\int_{0}^{x} f(s) d s\right)^{p} d x \leq\left(\frac{p}{\alpha-1}\right)^{p} \int_{0}^{\infty} \frac{1}{x^{\alpha-p}} f^{p}(x) d x
$$

(ii) If $p>1$ and $\alpha<1$, then

$$
\int_{0}^{\infty} \frac{1}{x^{\alpha}}\left(\int_{x}^{\infty} f(s) d s\right)^{p} d x \leq\left(\frac{p}{1-\alpha}\right)^{p} \int_{0}^{\infty} \frac{1}{x^{\alpha-p}} f^{p}(x) d x .
$$

In the same year, Copson [11] generalized the discrete Hardy inequality (1.1) and obtained the next two discrete inequalities.

Theorem 1.6 Let $\{a(n)\}_{n=1}^{\infty}$ and $\{g(n)\}_{n=1}^{\infty}$ be sequences of nonnegative real numbers. Then

$$
\begin{aligned}
& \sum_{n=1}^{\infty} \frac{g(n)\left(\sum_{m=1}^{n} g(m) a(m)\right)^{p}}{\left(\sum_{m=1}^{n} g(m)\right)^{\alpha}} \leq\left(\frac{p}{\alpha-1}\right)^{p} \sum_{n=1}^{\infty} g(n) a^{p}(n)\left(\sum_{m=1}^{n} g(m)\right)^{p-\alpha}, \\
& \quad \text { for } p \geq \alpha>1,
\end{aligned}
$$


and

$$
\begin{aligned}
& \sum_{n=1}^{\infty} \frac{g(n)\left(\sum_{m=n}^{\infty} g(m) a(m)\right)^{p}}{\left(\sum_{m=1}^{n} g(m)\right)^{\alpha}} \leq\left(\frac{p}{1-\alpha}\right)^{p} \sum_{n=1}^{\infty} g(n) a^{p}(n)\left(\sum_{m=1}^{n} g(m)\right)^{p-\alpha}, \\
& \quad \text { for } p>1>\alpha \geq 0 .
\end{aligned}
$$

In 1970, Leindler [22] studied the case that the sum $\sum_{n=1}^{\infty} g(m)<\infty$ on the left-hand side of inequality (1.8) is replaced with the sum $\sum_{m=n}^{\infty} g(m)<\infty$. His result can be written as:

Theorem 1.7 Let $\{a(n)\}_{n=1}^{\infty}$ and $\{g(n)\}_{n=1}^{\infty}$ be sequences of nonnegative real numbers with $\sum_{m=n}^{\infty} g(m)<\infty$. If $p>1>\alpha \geq 0$, then

$$
\sum_{n=1}^{\infty} \frac{g(n)\left(\sum_{m=1}^{n} g(m) a(m)\right)^{p}}{\left(\sum_{m=n}^{\infty} g(m)\right)^{\alpha}} \leq\left(\frac{p}{1-\alpha}\right)^{p} \sum_{n=1}^{\infty} g(n) a^{p}(n)\left(\sum_{m=n}^{\infty} g(m)\right)^{p-\alpha}
$$

In 1976, Copson [12] gave the continuous versions of inequalities (1.8) and (1.9). Specifically, he established the following result.

Theorem 1.8 Let $f$ and $g$ be nonnegative continuous functions on $[0, \infty)$. Then

$$
\begin{aligned}
& \int_{0}^{\infty} \frac{g(x)\left(\int_{0}^{x} g(s) f(s) d s\right)^{p}}{\left(\int_{0}^{x} g(s) d s\right)^{\alpha}} d x \leq\left(\frac{p}{\alpha-1}\right)^{p} \int_{0}^{\infty} g(x) f^{p}(x)\left(\int_{0}^{x} g(s) d s\right)^{p-\alpha} d x, \\
& \quad \text { for } p \geq \alpha>1,
\end{aligned}
$$

and

$$
\begin{aligned}
& \int_{0}^{\infty} \frac{g(x)\left(\int_{x}^{\infty} g(s) f(s) d s\right)^{p}}{\left(\int_{0}^{x} g(s) d s\right)^{\alpha}} d x \leq\left(\frac{p}{1-\alpha}\right)^{p} \int_{0}^{\infty} g(x) f^{p}(x)\left(\int_{0}^{x} g(s) d s\right)^{p-\alpha} d x, \\
& \quad \text { for } p>1>\alpha \geq 0 .
\end{aligned}
$$

In 1987, Bennett [5], similarly to what Leindler did in Theorem 1.7, proved the following result.

Theorem 1.9 Let $\{a(n)\}_{n=1}^{\infty}$ and $\{g(n)\}_{n=1}^{\infty}$ be sequences of nonnegative real numbers with $\sum_{m=n}^{\infty} g(m)<\infty$. If $p \geq \alpha>1$, then

$$
\sum_{n=1}^{\infty} \frac{g(n)\left(\sum_{m=n}^{\infty} g(m) a(m)\right)^{p}}{\left(\sum_{m=n}^{\infty} g(m)\right)^{\alpha}} \leq\left(\frac{p}{\alpha-1}\right)^{p} \sum_{n=1}^{\infty} g(n) a^{p}(n)\left(\sum_{m=n}^{\infty} g(m)\right)^{p-\alpha}
$$

The study of Hardy-type inequalities attracted and still attracts the attention of many researchers. Over several decades many generalizations, extensions, and refinements have been made to the above inequalities; we refer the interested reader to the papers $[3-7,11$, $14,15,17,22]$, the books $[16,20,21,26]$ and the references cited therein.

The theory of time scales, which has recently received a lot of attention, was initiated by Stefan Hilger in his PhD thesis in order to unify discrete and continuous analysis [18]. The general idea is to prove a result for a dynamic equation or a dynamic inequality where 
the domain of the unknown function is a so-called time scale $\mathbb{T}$, which may be an arbitrary closed subset of the real numbers $\mathbb{R}$, see $[8,10]$. The three most popular examples of calculus on time scales are differential calculus, difference calculus, and quantum calculus (see [19]), i.e., when $\mathbb{T}=\mathbb{R}, \mathbb{T}=\mathbb{Z}$, and $\mathbb{T}=\overline{q^{\mathbb{Z}}}=\left\{q^{z}: z \in \mathbb{Z}\right\} \cup\{0\}$ where $q>1$. The book on the subject of time scales by Bohner and Peterson [9] summarizes and organizes much of time scales calculus. During the past decade, a number of dynamic inequalities have been established by some authors who were motivated by some applications, for example, when studying the behavior of solutions of certain class of dynamic equations on a time scale $\mathbb{T}$, see $[2,9,23,24]$ and the references cited therein.

In 2005, К̌ehák [27] was the first in extending Hardy-type inequalities to time scales. In particular, he generalized inequalities (1.1) and (1.2) to an arbitrary time scale and applied his results in the oscillation theory of half-linear dynamic equations, in doing so, he unified them in one form as shown next.

Theorem 1.10 Let $\mathbb{T}$ be a time scale, and $f \in C_{r d}\left([a, \infty)_{\mathbb{T}},[0, \infty)\right)$. If $p>1$, then

$$
\int_{a}^{\infty}\left(\frac{\int_{a}^{\sigma(t)} f(s) \Delta s}{\sigma(t)-a}\right)^{p} \Delta t<\left(\frac{p}{p-1}\right)^{p} \int_{a}^{\infty} f^{p}(t) \Delta t,
$$

unless $f \equiv 0$.

Furthermore, if $\mu(t) / t \rightarrow 0$ as $t \rightarrow \infty$, then inequality (1.14) is sharp.

In 2014, Saker et al. [28] proved the following four dynamic inequalities of Hardy-type.

Theorem 1.11 Let $\mathbb{T}$ be a time scale with $a \in[0, \infty)_{\mathbb{T}}$. In addition, let $f$ and $g$ be nonnegative rd-continuous functions on $[a, \infty)_{\mathbb{T}}$.

(i) If $p \geq \alpha>1$, then

$$
\begin{aligned}
& \int_{a}^{\infty} \frac{g(t)\left(\int_{a}^{\sigma(t)} g(s) f(s) \Delta s\right)^{p}}{\left(\int_{a}^{\sigma(t)} g(s) \Delta s\right)^{\alpha}} \Delta t \\
& \quad \leq\left(\frac{p}{\alpha-1}\right)^{p} \int_{a}^{\infty} \frac{g(t) f^{p}(t)\left(\int_{a}^{\sigma(t)} g(s) \Delta s\right)^{\alpha(p-1)}}{\left(\int_{a}^{t} g(s) \Delta s\right)^{p(\alpha-1)}} \Delta t .
\end{aligned}
$$

(ii) If $p>1>\alpha \geq 0$, then

$$
\begin{aligned}
& \int_{a}^{\infty} \frac{g(t)\left(\int_{t}^{\infty} g(s) f(s) \Delta s\right)^{p}}{\left(\int_{a}^{\sigma(t)} g(s) \Delta s\right)^{\alpha}} \Delta t \\
& \quad \leq\left(\frac{p}{1-\alpha}\right)^{p} \int_{a}^{\infty} g(t) f^{p}(t)\left(\int_{a}^{\sigma(t)} g(s) \Delta s\right)^{p-\alpha} \Delta t .
\end{aligned}
$$

(iii) If $p>1>\alpha \geq 0$, then

$$
\begin{aligned}
& \int_{a}^{\infty} \frac{g(t)\left(\int_{a}^{\sigma(t)} g(s) f(s) \Delta s\right)^{p}}{\left(\int_{t}^{\infty} g(s) \Delta s\right)^{\alpha}} \Delta t \\
& \quad \leq\left(\frac{p}{1-\alpha}\right)^{p} \int_{a}^{\infty} g(t) f^{p}(t)\left(\int_{t}^{\infty} g(s) \Delta s\right)^{p-\alpha} \Delta t .
\end{aligned}
$$


(iv) If $p \geq \alpha>1$, then

$$
\begin{aligned}
& \int_{a}^{\infty} \frac{g(t)\left(\int_{t}^{\infty} g(s) f(s) \Delta s\right)^{p}}{\left(\int_{t}^{\infty} g(s) \Delta s\right)^{\alpha}} \Delta t \\
& \quad \leq\left(\frac{p}{\alpha-1}\right)^{p} \int_{a}^{\infty} g(t) f^{p}(t)\left(\int_{t}^{\infty} g(s) \Delta s\right)^{p-\alpha} \Delta t .
\end{aligned}
$$

Note that inequality (1.15) unifies inequalities (1.8) and (1.11) in one form by giving their time scales version. Similarly, inequality (1.16) gives the time scales version of inequalities (1.9) and (1.12), inequality (1.17) gives the time scales version of (1.10), and (1.18) gives the time scales version of inequality (1.13).

In this paper, we will establish some original dynamic inequalities of Hardy-type on time scales which may be considered as generalizations of inequalities (1.15), (1.16), (1.17), and (1.18). The obtained results will extend some known Hardy-type integral inequalities, unify and extend some continuous inequalities and their corresponding discrete analogues. The paper is arranged as follows: In Sect. 2, some basic concepts of the calculus on time scales and useful lemmas are introduced. In Sect. 3, we state and prove the main results. Section 4 concludes the manuscript.

\section{Preliminaries and lemmas on time scales}

A time scale $\mathbb{T}$ is an arbitrary nonempty closed subset of the real numbers. We assume throughout that $\mathbb{T}$ has the topology that it inherits from the standard topology on the real numbers $\mathbb{R}$. We define the forward jump operator $\sigma: \mathbb{T} \rightarrow \mathbb{T}$ by

$$
\sigma(t):=\inf \{s \in \mathbb{T}: s>t\}, \quad t \in \mathbb{T} .
$$

In the previous definition, we set $\inf \emptyset=\sup \mathbb{T}$ (i.e., if $t$ is the maximum of $\mathbb{T}$, then $\sigma(t)=$ $t$ ), where $\emptyset$ is the empty set.

Let $f: \mathbb{T} \rightarrow \mathbb{R}$ be a real-valued function on a time scale $\mathbb{T}$. Then, for $t \in \mathbb{T}^{\kappa}$, we define $f^{\Delta}(t)$ to be the number (if it exists) with the property that given any $\varepsilon>0$ there is a neighborhood $U$ of $t$ such that, for all $s \in U$, we have

$$
\left|[f(\sigma(t))-f(s)]-f^{\Delta}(t)[\sigma(t)-s]\right| \leq \varepsilon|\sigma(t)-s|
$$

In this case, we say that $f$ is delta differentiable on $\mathbb{T}^{\kappa}$ provided $f^{\Delta}(t)$ exists for all $t \in \mathbb{T}^{\kappa}$.

If $f$ and $g$ are two delta differentiable functions, then the product rule for the derivative of $f g$ is given as follows:

$$
(f g)^{\Delta}(t)=f^{\Delta}(t) g(t)+f(\sigma(t)) g^{\Delta}(t)=f(t) g^{\Delta}(t)+f^{\Delta}(t) g(\sigma(t)) .
$$

The formula for the delta integration by parts on time scales can be written as

$$
\int_{a}^{b} u(t) z^{\Delta}(t) \Delta t=[u(t) z(t)]_{a}^{b}-\int_{a}^{b} u^{\Delta}(t) z^{\sigma}(t) \Delta t
$$

We will frequently use the following important relations. 
(i) If $\mathbb{T}=\mathbb{R}$, then

$$
\sigma(t)=t, \quad f^{\Delta}(t)=f^{\prime}(t), \quad \int_{a}^{b} f(t) \Delta t=\int_{a}^{b} f(t) d t
$$

(ii) If $\mathbb{T}=\mathbb{Z}$, then

$$
\sigma(t)=t+1, \quad f^{\Delta}(t)=\Delta f(t), \quad \int_{a}^{b} f(t) \Delta t=\sum_{t=a}^{b-1} f(t)
$$

(iii) If $\mathbb{T}=h \mathbb{Z}$, then

$$
\sigma(t)=t+h, \quad f^{\Delta}(t)=\frac{f(t+h)-f(t)}{h}, \quad \int_{a}^{b} f(t) \Delta t=\sum_{t=\frac{a}{h}}^{\frac{b}{h}-1} h f(h t) .
$$

(iv) If $\mathbb{T}=\overline{q^{\mathbb{Z}}}$, then

$$
\begin{aligned}
& \sigma(t)=q t, \quad f^{\Delta}(t)=\frac{f(q t)-f(t)}{(q-1) t}, \\
& \int_{a}^{b} f(t) \Delta t=(q-1) \sum_{t=\log _{q} a}^{\left(\log _{q} b\right)-1} q^{t} f\left(q^{t}\right) .
\end{aligned}
$$

Lemma 2.1 (Chain Rule on Time Scales, see [9]) Assume $g: \mathbb{R} \rightarrow \mathbb{R}$, is continuous function $g: \mathbb{T} \rightarrow \mathbb{R}$ is delta differentiable on $\mathbb{T}^{\kappa}$, and $f: \mathbb{R} \rightarrow \mathbb{R}$ is continuously differentiable. Then there exists $c \in[t, \sigma(t)]$ with

$$
(f \circ g)^{\Delta}(t)=f^{\prime}(g(c)) g^{\Delta}(t)
$$

Lemma 2.2 (Dynamic Hölder Inequality, see [1]) Let $a, b \in \mathbb{T}$ and $f, g \in C_{r d}\left([a, b]_{\mathbb{T}}\right.$, $[0, \infty))$. If $p, q>1$ with $1 / p+1 / q=1$, then

$$
\int_{a}^{b} f(t) g(t) \Delta t \leq\left(\int_{a}^{b} f^{p}(t) \Delta t\right)^{1 / p}\left(g^{q}(t) \Delta t\right)^{1 / q}
$$

\section{Main results}

Theorem 3.1 Let $\mathbb{T}$ be a time scale with $a \in[0, \infty)_{\mathbb{T}}$. In addition, let $f, g, k, r, w, v$ be nonnegative rd-continuous functions on $[a, \infty)_{\mathbb{T}}$ such that $k$ is nonincreasing. Further, assume there exist $\theta, \beta \geq 0$ such that $\frac{w^{\Delta}(t)}{w(t)} \leq \theta\left(\frac{G^{\Delta}(t)}{G^{\sigma}(t)}\right)$ and $\frac{v^{\Delta}(t)}{v^{\sigma}(t)} \leq \beta\left(\frac{K^{\Delta}(t)}{K(t)}\right)$, where

$$
G(t)=\int_{a}^{t} g(s) \Delta s \quad \text { with } G(\infty)=\infty \quad \text { and } \quad K(t)=\int_{a}^{t} r(s) f(s) \Delta s, \quad t \in[a, \infty)_{\mathbb{T}} .
$$

If $p \geq 1$ and $\alpha>\theta+1$, then

$$
\begin{aligned}
& \int_{a}^{\infty} k^{\sigma}(t) v^{\sigma}(t) w(t) g(t)\left(G^{\sigma}(t)\right)^{-\alpha}\left(K^{\sigma}(t)\right)^{p} \Delta t \\
& \quad \leq\left(\frac{p+\beta}{\alpha-\theta-1}\right)^{p} \int_{a}^{\infty} \frac{k^{\sigma}(t) v^{\sigma}(t) w(t) r^{p}(t) f^{p}(t)\left(G^{\sigma}(t)\right)^{\alpha(p-1)}}{g^{p-1}(t) G^{p(\alpha-1)}(t)} \Delta t .
\end{aligned}
$$


Proof Using integration by parts formula on time scales (2.2) with

$$
u^{\Delta}(t)=w(t) g(t)\left(G^{\sigma}(t)\right)^{-\alpha} \quad \text { and } \quad z^{\sigma}(t)=k^{\sigma}(t) v^{\sigma}(t)\left(K^{\sigma}(t)\right)^{p}
$$

we have

$$
\begin{aligned}
& \int_{a}^{\infty} k^{\sigma}(t) v^{\sigma}(t) w(t) g(t)\left(G^{\sigma}(t)\right)^{-\alpha}\left(K^{\sigma}(t)\right)^{p} \Delta t \\
& \quad=\left[u(t) k(t) v(t) K^{p}(t)\right]_{a}^{\infty}+\int_{a}^{\infty}(-u(t))\left(k(t) v(t) K^{p}(t)\right)^{\Delta} \Delta t,
\end{aligned}
$$

where we assumed that

$$
u(t)=-\int_{t}^{\infty} w(s) g(s)\left(G^{\sigma}(s)\right)^{-\alpha} \Delta s .
$$

Using the chain rule on time scales (2.7), the product rule (2.1), and the hypothesis $\frac{w^{\Delta}(t)}{w(t)} \leq$ $\theta\left(\frac{G^{\Delta}(t)}{G^{\sigma}(t)}\right)$, we see that there is $c \in[s, \sigma(s)]$ such that

$$
\begin{aligned}
\left(w(s) G^{1-\alpha}(s)\right)^{\Delta} & =w^{\Delta}(s)\left(G^{\sigma}(s)\right)^{1-\alpha}+w(s)\left(G^{1-\alpha}(s)\right)^{\Delta} \\
& \leq \theta w(s) G^{\Delta}(s)\left(G^{\sigma}(s)\right)^{-\alpha}+(1-\alpha) w(s) G^{-\alpha}(c) G^{\Delta}(s) .
\end{aligned}
$$

Since $G^{\Delta}(s)=g(s) \geq 0, c \leq \sigma(s)$ and $\alpha>1$, we get

$$
\begin{aligned}
\left(w(s) G^{1-\alpha}(s)\right)^{\Delta} & \leq \theta w(s) g(s)\left(G^{\sigma}(s)\right)^{-\alpha}+(1-\alpha) w(s) g(s)\left(G^{\sigma}(s)\right)^{-\alpha} \\
& =(1-\alpha+\theta) w(s) g(s)\left(G^{\sigma}(s)\right)^{-\alpha} .
\end{aligned}
$$

This gives us that

$$
w(s) g(s)\left(G^{\sigma}(s)\right)^{-\alpha} \leq \frac{1}{1-\alpha+\theta}\left(w(s) G^{1-\alpha}(s)\right)^{\Delta} .
$$

Hence

$$
\begin{aligned}
-u(t) & =\int_{t}^{\infty} w(s) g(s)\left(G^{\sigma}(s)\right)^{-\alpha} \Delta s \leq \frac{1}{1-\alpha+\theta} \int_{t}^{\infty}\left(w(s) G^{1-\alpha}(s)\right)^{\Delta} \Delta s \\
& =\frac{1}{\alpha-\theta-1} w(t) G^{1-\alpha}(t) .
\end{aligned}
$$

Applying the product rule (2.1) and the chain rule on time scales (2.7), we see that there exists $c \in[t, \sigma(t)]$ such that

$$
\begin{aligned}
\left(k(t) v(t) K^{p}(t)\right)^{\Delta} & =(k(t) v(t))^{\Delta} K^{p}(t)+k^{\sigma}(t) v^{\sigma}(t)\left(K^{p}(t)\right)^{\Delta} \\
& =k^{\Delta}(t) v(t) K^{p}(t)+k^{\sigma}(t) v^{\Delta}(t) K^{p}(t)+p k^{\sigma}(t) v^{\sigma}(t) K^{p-1}(c) K^{\Delta}(t) .
\end{aligned}
$$

Since $k^{\Delta}(t) \leq 0, K^{\Delta}(t)=r(t) f(t) \geq 0, c \leq \sigma(t), p \geq 1$, and $\frac{v^{\Delta}(t)}{v^{\sigma}(t)} \leq \beta\left(\frac{K^{\Delta}(t)}{K(t)}\right)$, we have

$$
\begin{aligned}
\left(k(t) v(t) K^{p}(t)\right)^{\Delta} & \leq \beta k^{\sigma}(t) v^{\sigma}(t) r(t) f(t) K^{p-1}(t)+p k^{\sigma}(t) v^{\sigma}(t) r(t) f(t)\left(K^{\sigma}(t)\right)^{p-1} \\
& \leq(p+\beta) k^{\sigma}(t) v^{\sigma}(t) r(t) f(t)\left(K^{\sigma}(t)\right)^{p-1}
\end{aligned}
$$


Combining (3.2), (3.3), and (3.4), we get (note that $K(a)=0$ and $u(\infty)=0$ )

$$
\begin{aligned}
& \int_{a}^{\infty} k^{\sigma}(t) v^{\sigma}(t) w(t) g(t)\left(G^{\sigma}(t)\right)^{-\alpha}\left(K^{\sigma}(t)\right)^{p} \Delta t \\
& \quad \leq \frac{p+\beta}{\alpha-\theta-1} \int_{a}^{\infty} k^{\sigma}(t) v^{\sigma}(t) w(t) r(t) f(t) G^{1-\alpha}(t)\left(K^{\sigma}(t)\right)^{p-1} \Delta t
\end{aligned}
$$

or equivalently,

$$
\begin{aligned}
\int_{a}^{\infty} & k^{\sigma}(t) v^{\sigma}(t) w(t) g(t)\left(G^{\sigma}(t)\right)^{-\alpha}\left(K^{\sigma}(t)\right)^{p} \Delta t \\
\leq & \frac{p+\beta}{\alpha-\theta-1} \int_{a}^{\infty}\left(\left(k^{\sigma}(t) v^{\sigma}(t) w(t) g(t)\right)^{(p-1) / p}\left(G^{\sigma}(t)\right)^{-\alpha(p-1) / p}\left(K^{\sigma}(t)\right)^{p-1}\right) \\
& \times\left(\frac{\left(k^{\sigma}(t) v^{\sigma}(t) w(t)\right)^{1 / p} r(t) f(t)\left(G^{\sigma}(t)\right)^{\alpha(p-1) / p}}{g^{(p-1) / p}(t) G^{\alpha-1}(t)}\right) \Delta t .
\end{aligned}
$$

Employing the dynamic Hölder inequality (2.8) with indices $p$ and $p /(p-1)$, we get

$$
\begin{aligned}
& \int_{a}^{\infty} k^{\sigma}(t) v^{\sigma}(t) w(t) g(t)\left(G^{\sigma}(t)\right)^{-\alpha}\left(K^{\sigma}(t)\right)^{p} \Delta t \\
& \leq \frac{p+\beta}{\alpha-\theta-1}\left(\int_{a}^{\infty} k^{\sigma}(t) v^{\sigma}(t) w(t) g(t)\left(G^{\sigma}(t)\right)^{-\alpha}\left(K^{\sigma}(t)\right)^{p} \Delta t\right)^{(p-1) / p} \\
& \quad \times\left(\int_{a}^{\infty} \frac{k^{\sigma}(t) v^{\sigma}(t) w(t) r^{p}(t) f^{p}(t)\left(G^{\sigma}(t)\right)^{\alpha(p-1)}}{g^{p-1}(t) G^{p(\alpha-1)}(t)} \Delta t\right)^{1 / p},
\end{aligned}
$$

which implies that

$$
\begin{aligned}
& \int_{a}^{\infty} k^{\sigma}(t) v^{\sigma}(t) w(t) g(t)\left(G^{\sigma}(t)\right)^{-\alpha}\left(K^{\sigma}(t)\right)^{p} \Delta t \\
& \quad \leq\left(\frac{p+\beta}{\alpha-\theta-1}\right)^{p} \int_{a}^{\infty} \frac{k^{\sigma}(t) v^{\sigma}(t) w(t) r^{p}(t) f^{p}(t)\left(G^{\sigma}(t)\right)^{\alpha(p-1)}}{g^{p-1}(t) G^{p(\alpha-1)}(t)} \Delta t .
\end{aligned}
$$

This completes the proof.

Remark 3.2 In Theorem 3.1, if we set $k(t)=v(t)=w(t)=1$ and $r(t)=g(t)$, then we may take $\theta=\beta=0$, and in this case inequality (3.1) reduces to inequality (1.15).

Corollary 3.3 If $\mathbb{T}=\mathbb{R}$ in Theorem 3.1, then, using relations (2.3), inequality (3.1) becomes

$$
\begin{aligned}
& \int_{a}^{\infty} k(t) v(t) w(t) g(t) G^{-\alpha}(t) K^{p}(t) d t \\
& \quad \leq\left(\frac{p+\beta}{\alpha-\theta-1}\right)^{p} \int_{a}^{\infty} \frac{k(t) v(t) w(t) r^{p}(t) f^{p}(t) G^{p-\alpha}(t)}{g^{p-1}(t)} d t
\end{aligned}
$$

where

$$
G(t)=\int_{a}^{t} g(s) d s \quad \text { and } \quad K(t)=\int_{a}^{t} r(s) f(s) d s .
$$


Remark 3.4 In Corollary 3.3, if we set $k(t)=v(t)=w(t)=1, r(t)=g(t)$ and $a=0$, then we may take $\theta=\beta=0$, and in this case inequality (3.5) reduces to inequality (1.11).

Remark 3.5 In Corollary 3.3, if we set $k(t)=v(t)=w(t)=r(t)=g(t)=1$ and $a=0$, then we may take $\theta=\beta=0$, and in this case inequality (3.5) reduces to inequality (1.6).

Remark 3.6 In Corollary 3.3, if we set $k(t)=v(t)=w(t)=r(t)=g(t)=1, a=0$ and $p=\alpha$, then we may take $\theta=\beta=0$, and in this case inequality (3.5) reduces to the classical integral Hardy inequality (1.2).

Corollary 3.7 If $\mathbb{T}=h \mathbb{Z}$ in Theorem 3.1, then, using relations (2.5), inequality (3.1) becomes

$$
\begin{aligned}
& \sum_{t=\frac{a}{h}}^{\infty} k(h t+h) v(h t+h) w(h t) g(h t) G^{-\alpha}(h t+h) K^{p}(h t+h) \\
& \quad \leq\left(\frac{p+\beta}{\alpha-\theta-1}\right)^{p} \sum_{t=\frac{a}{h}}^{\infty} \frac{k(h t+h) v(h t+h) w(h t) r^{p}(h t) f^{p}(h t) G^{\alpha(p-1)}(h t+h)}{g^{p-1}(h t) G^{p(\alpha-1)}(h t)},
\end{aligned}
$$

where

$$
G(t)=h \sum_{s=\frac{a}{h}}^{\frac{t}{h}-1} g(h s) \quad \text { and } \quad K(t)=h \sum_{s=\frac{a}{h}}^{\frac{t}{h}-1} r(h s) f(h s) .
$$

Corollary 3.8 For $\mathbb{T}=\mathbb{Z}$, we simply take $h=1$ in Corollary 3.7. In this case, inequality (3.1) becomes

$$
\begin{aligned}
& \sum_{t=a}^{\infty} k(t+1) v(t+1) w(t) g(t) G^{-\alpha}(t+1) K^{p}(t+1) \\
& \quad \leq\left(\frac{p+\beta}{\alpha-\theta-1}\right)^{p} \sum_{t=a}^{\infty} \frac{k(t+1) v(t+1) w(t) r^{p}(t) f^{p}(t) G^{\alpha(p-1)}(t+1)}{g^{p-1}(t) G^{p(\alpha-1)}(t)}
\end{aligned}
$$

where

$$
G(t)=\sum_{s=a}^{t-1} g(s) \quad \text { and } \quad K(t)=\sum_{s=a}^{t-1} r(s) f(s)
$$

Remark 3.9 In Corollary 3.8, if we set $k(t)=v(t)=w(t)=1, r(t)=g(t)$ and $a=1$, then we may take $\theta=\beta=0$, and in this case inequality (3.7) reduces to

$$
\sum_{t=1}^{\infty} \frac{g(t)\left(\sum_{s=1}^{t} g(s) f(s)\right)^{p}}{\left(\sum_{s=1}^{t} g(s)\right)^{\alpha}} \leq\left(\frac{p}{\alpha-1}\right)^{p} \sum_{t=1}^{\infty} \frac{g(t) f^{p}(t)\left(\sum_{s=1}^{t} g(s)\right)^{\alpha(p-1)}}{\left(\sum_{s=1}^{t-1} g(s)\right)^{p(\alpha-1)}}
$$

which is another form of the discrete inequality (1.8). 
Corollary 3.10 If $\mathbb{T}=\overline{q^{\mathbb{Z}}}$ in Theorem 3.1, then, using relations (2.6), inequality (3.1) becomes

$$
\begin{aligned}
& \sum_{t=\log _{q} a}^{\infty} k\left(q^{t+1}\right) v\left(q^{t+1}\right) w\left(q^{t}\right) g\left(q^{t}\right) G^{-\alpha}\left(q^{t+1}\right) K^{p}\left(q^{t+1}\right) \\
& \leq\left(\frac{p+\beta}{\alpha-\theta-1}\right)^{p} \sum_{t=\log _{q} a}^{\infty} \frac{k\left(q^{t+1}\right) v\left(q^{t+1}\right) w\left(q^{t}\right) r^{p}\left(q^{t}\right) f^{p}\left(q^{t}\right) G^{\alpha(p-1)}\left(q^{t+1}\right)}{g^{p-1}\left(q^{t}\right) G^{p(\alpha-1)}\left(q^{t}\right)}
\end{aligned}
$$

where

$$
G(t)=(q-1) \sum_{s=\log _{q} a}^{\left(\log _{q} t\right)-1} q^{s} g\left(q^{s}\right) \quad \text { and } \quad K(t)=(q-1) \sum_{s=\log _{q} a}^{\left(\log _{q} t\right)-1} q^{s} r\left(q^{s}\right) f\left(q^{s}\right)
$$

Theorem 3.11 Suppose $\mathbb{T}$ is a time scale with $a \in[0, \infty)_{\mathbb{T}}$. Further, assume $f, g, k, r, w, v$ are nonnegative rd-continuous functions on $[a, \infty)_{\mathbb{T}}$ such that $k$ is nondecreasing. Moreover, suppose there exist $\theta, \beta \geq 0$ such that $\frac{w^{\Delta}(t)}{w^{\sigma}(t)} \geq \theta\left(\frac{G^{\Delta}(t)}{G(t)}\right)$ and $\frac{v^{\Delta}(t)}{v(t)} \geq \beta\left(\frac{F^{\Delta}(t)}{F^{\sigma}(t)}\right)$, where

$$
G(t)=\int_{a}^{t} g(s) \Delta s \quad \text { with } G(\infty)=\infty, \quad \text { and } \quad F(t)=\int_{t}^{\infty} r(s) f(s) \Delta s, \quad t \in[a, \infty)_{\mathbb{T}} .
$$

If $p \geq 1$ and $0 \leq \alpha<1$, then

$$
\begin{aligned}
& \int_{a}^{\infty} k(t) v(t) w^{\sigma}(t) g(t)\left(G^{\sigma}(t)\right)^{-\alpha} F^{p}(t) \Delta t \\
& \quad \leq\left(\frac{p+\beta}{1-\alpha+\theta}\right)^{p} \int_{a}^{\infty} \frac{k(t) v(t) w^{\sigma}(t) r^{p}(t) f^{p}(t)\left(G^{\sigma}(t)\right)^{p-\alpha}}{g^{p-1}(t)} \Delta t .
\end{aligned}
$$

Proof From integration by parts formula on time scales (2.2), we have

$$
\begin{aligned}
& \int_{a}^{\infty} k(t) v(t) w^{\sigma}(t) g(t)\left(G^{\sigma}(t)\right)^{-\alpha} F^{p}(t) \Delta t \\
& \quad=\left[u(t) k(t) v(t) F^{p}(t)\right]_{a}^{\infty}+\int_{a}^{\infty} u^{\sigma}(t)\left(-k(t) v(t) F^{p}(t)\right)^{\Delta} \Delta t,
\end{aligned}
$$

where

$$
u(t)=\int_{a}^{t} w^{\sigma}(s) g(s)\left(G^{\sigma}(s)\right)^{-\alpha} \Delta s
$$

Employing the chain rule on time scales (2.7), the product rule (2.1), and the assumption $\frac{w^{\Delta}(t)}{w^{\sigma}(t)} \geq \theta\left(\frac{G^{\Delta}(t)}{G(t)}\right)$, we see that there is $c \in[s, \sigma(s)]$ such that

$$
\begin{aligned}
\left(w(s) G^{1-\alpha}(s)\right)^{\Delta} & =w^{\Delta}(s) G^{1-\alpha}(s)+w^{\sigma}(s)\left(G^{1-\alpha}(s)\right)^{\Delta} \\
& \geq \theta w^{\sigma}(s) G^{-\alpha}(s) G^{\Delta}(s)+(1-\alpha) w^{\sigma}(s) G^{-\alpha}(c) G^{\Delta}(s) .
\end{aligned}
$$


As $G^{\Delta}(s)=g(s) \geq 0, c \leq \sigma(s)$ and $0 \leq \alpha<1$, we get

$$
\begin{aligned}
\left(w(s) G^{1-\alpha}(s)\right)^{\Delta} & \geq \theta w^{\sigma}(s) g(s)\left(G^{\sigma}(s)\right)^{-\alpha}+(1-\alpha) w^{\sigma}(s) g(s)\left(G^{\sigma}(s)\right)^{-\alpha} \\
& =(1-\alpha+\theta) w^{\sigma}(s) g(s)\left(G^{\sigma}(s)\right)^{-\alpha},
\end{aligned}
$$

which implies

$$
w^{\sigma}(s) g(s)\left(G^{\sigma}(s)\right)^{-\alpha} \leq \frac{1}{1-\alpha+\theta}\left(w(s) G^{1-\alpha}(s)\right)^{\Delta} .
$$

Therefore

$$
\begin{aligned}
u^{\sigma}(t) & =\int_{a}^{\sigma(t)} w^{\sigma}(s) g(s)\left(G^{\sigma}(s)\right)^{-\alpha} \Delta s \leq \frac{1}{1-\alpha+\theta} \int_{a}^{\sigma(t)}\left(w(s) G^{1-\alpha}(s)\right)^{\Delta} \Delta s \\
& =\frac{1}{1-\alpha+\theta} w^{\sigma}(t)\left(G^{\sigma}(t)\right)^{1-\alpha} .
\end{aligned}
$$

Utilizing the product rule (2.1) and the chain rule on time scales (2.7), we see that there exists $c \in[t, \sigma(t)]$ such that

$$
\begin{aligned}
\left(-k(t) v(t) F^{p}(t)\right)^{\Delta} & =-\left((k(t) v(t))^{\Delta}\left(F^{\sigma}(t)\right)^{p}+k(t) v(t)\left(F^{p}(t)\right)^{\Delta}\right) \\
& =-\left(k^{\Delta}(t) v^{\sigma}(t)\left(F^{\sigma}(t)\right)^{p}+k(t) v^{\Delta}(t)\left(F^{\sigma}(t)\right)^{p}+p k(t) v(t) F^{p-1}(c) F^{\Delta}(t)\right) .
\end{aligned}
$$

Since $k^{\Delta}(t) \geq 0, F^{\Delta}(t)=-r(t) f(t) \leq 0, c \geq t, p \geq 1$, and $\frac{v^{\Delta}(t)}{v(t)} \geq \beta\left(\frac{F^{\Delta}(t)}{F^{\sigma}(t)}\right)$, we get

$$
\begin{aligned}
\left(-k(t) v(t) F^{p}(t)\right)^{\Delta} & \leq \beta k(t) v(t) r(t) f(t)\left(F^{\sigma}(t)\right)^{p-1}+p k(t) v(t) r(t) f(t) F^{p-1}(t) \\
& \leq(p+\beta) k(t) v(t) r(t) f(t) F^{p-1}(t) .
\end{aligned}
$$

From (3.11), (3.12), and (3.13), we get (note that $F(\infty)=0$ and $u(a)=0$ )

$$
\begin{aligned}
& \int_{a}^{\infty} k(t) v(t) w^{\sigma}(t) g(t)\left(G^{\sigma}(t)\right)^{-\alpha} F^{p}(t) \Delta t \\
& \quad \leq \frac{p+\beta}{1-\alpha+\theta} \int_{a}^{\infty} k(t) v(t) w^{\sigma}(t) r(t) f(t)\left(G^{\sigma}(t)\right)^{1-\alpha} F^{p-1}(t) \Delta t
\end{aligned}
$$

which is equivalent to

$$
\begin{aligned}
\int_{a}^{\infty} & k(t) v(t) w^{\sigma}(t) g(t)\left(G^{\sigma}(t)\right)^{-\alpha} F^{p}(t) \Delta t \\
\leq & \frac{p+\beta}{1-\alpha+\theta} \int_{a}^{\infty}\left(\left(k(t) v(t) w^{\sigma}(t) g(t)\right)^{(p-1) / p}\left(G^{\sigma}(t)\right)^{-\alpha(p-1) / p} F^{p-1}(t)\right) \\
& \times\left(\frac{\left(k(t) v(t) w^{\sigma}(t)\right)^{1 / p} r(t) f(t)\left(G^{\sigma}(t)\right)^{(p-\alpha) / p}}{g^{(p-1) / p}(t)}\right) \Delta t .
\end{aligned}
$$


Applying the dynamic Hölder inequality (2.8) with indices $p$ and $p /(p-1)$, we obtain

$$
\begin{aligned}
& \int_{a}^{\infty} k(t) v(t) w^{\sigma}(t) g(t)\left(G^{\sigma}(t)\right)^{-\alpha} F^{p}(t) \Delta t \\
& \leq \frac{p+\beta}{1-\alpha+\theta}\left(\int_{a}^{\infty} k(t) v(t) w^{\sigma}(t) g(t)\left(G^{\sigma}(t)\right)^{-\alpha} F^{p}(t) \Delta t\right)^{(p-1) / p} \\
& \quad \times\left(\int_{a}^{\infty} \frac{k(t) v(t) w^{\sigma}(t) r^{p}(t) f^{p}(t)\left(G^{\sigma}(t)\right)^{p-\alpha}}{g^{p-1}(t)} \Delta t\right)^{1 / p}
\end{aligned}
$$

This leads to

$$
\begin{aligned}
& \int_{a}^{\infty} k(t) v(t) w^{\sigma}(t) g(t)\left(G^{\sigma}(t)\right)^{-\alpha} F^{p}(t) \Delta t \\
& \quad \leq\left(\frac{p+\beta}{1-\alpha+\theta}\right)^{p} \int_{a}^{\infty} \frac{k(t) v(t) w^{\sigma}(t) r^{p}(t) f^{p}(t)\left(G^{\sigma}(t)\right)^{p-\alpha}}{g^{p-1}(t)} \Delta t
\end{aligned}
$$

which is our desired result.

Remark 3.12 In Theorem 3.11, if we set $k(t)=v(t)=w(t)=1$ and $r(t)=g(t)$, then we may take $\theta=\beta=0$, and in this case inequality (3.10) reduces to inequality (1.16).

Corollary 3.13 If $\mathbb{T}=\mathbb{R}$ in Theorem 3.11, then, using relations (2.3), inequality (3.10) becomes

$$
\begin{aligned}
& \int_{a}^{\infty} k(t) v(t) w(t) g(t) G^{-\alpha}(t) F^{p}(t) d t \\
& \quad \leq\left(\frac{p+\beta}{1-\alpha+\theta}\right)^{p} \int_{a}^{\infty} \frac{k(t) v(t) w(t) r^{p}(t) f^{p}(t) G^{p-\alpha}(t)}{g^{p-1}(t)} d t
\end{aligned}
$$

where

$$
G(t)=\int_{a}^{t} g(s) d s \text { and } \quad F(t)=\int_{t}^{\infty} r(s) f(s) d s
$$

Remark 3.14 In Corollary 3.13, if we set $k(t)=v(t)=w(t)=1, r(t)=g(t)$ and $a=0$, then we may take $\theta=\beta=0$, and in this case inequality (3.14) reduces to inequality (1.12).

Remark 3.15 In Corollary 3.13, if we set $k(t)=v(t)=w(t)=r(t)=g(t)=1$ and $a=0$, then we may take $\theta=\beta=0$, and in this case inequality (3.14) reduces to inequality (1.7).

Remark 3.16 In Corollary 3.13, if we set $k(t)=v(t)=w(t)=r(t)=g(t)=1, a=0$ and $\alpha=0$, then we may take $\theta=\beta=0$, and in this case inequality (3.14) reduces to inequality (1.5). 
Corollary 3.17 If $\mathbb{T}=h \mathbb{Z}$ in Theorem 3.11, then, using relations (2.5), inequality (3.10) becomes

$$
\begin{aligned}
& \sum_{t=\frac{a}{h}}^{\infty} k(h t) v(h t) w(h t+h) g(h t) G^{-\alpha}(h t+h) F^{p}(h t) \\
& \quad \leq\left(\frac{p+\beta}{1-\alpha+\theta}\right)^{p} \sum_{t=\frac{a}{h}}^{\infty} \frac{k(h t) v(h t) w(h t+h) r^{p}(h t) f^{p}(h t) G^{p-\alpha}(h t+h)}{g^{p-1}(h t)},
\end{aligned}
$$

where

$$
G(t)=h \sum_{s=\frac{a}{h}}^{\frac{t}{h}-1} g(h s) \quad \text { and } \quad F(t)=h \sum_{s=\frac{t}{h}}^{\infty} r(h s) f(h s) .
$$

Corollary 3.18 For $\mathbb{T}=\mathbb{Z}$, we simply take $h=1$ in Corollary 3.17. In this case, inequality (3.10) becomes

$$
\begin{aligned}
& \sum_{t=a}^{\infty} k(t) v(t) w(t+1) g(t) G^{-\alpha}(t+1) F^{p}(t) \\
& \quad \leq\left(\frac{p+\beta}{1-\alpha+\theta}\right)^{p} \sum_{t=a}^{\infty} \frac{k(t) v(t) w(t+1) r^{p}(t) f^{p}(t) G^{p-\alpha}(t+1)}{g^{p-1}(t)},
\end{aligned}
$$

where

$$
G(t)=\sum_{s=a}^{t-1} g(s) \quad \text { and } \quad F(t)=\sum_{s=t}^{\infty} r(s) f(s) .
$$

Remark 3.19 In Corollary 3.18, if we set $k(t)=v(t)=w(t)=1, r(t)=g(t)$ and $a=1$, then we may take $\theta=\beta=0$, and in this case inequality (3.16) reduces to inequality (1.9).

Corollary 3.20 If $\mathbb{T}=\overline{q^{\mathbb{Z}}}$ in Theorem 3.11, then, using relations (2.6), inequality (3.10) becomes

$$
\begin{aligned}
& \sum_{t=\log _{q} a}^{\infty} k\left(q^{t}\right) v\left(q^{t}\right) w\left(q^{t+1}\right) g\left(q^{t}\right) G^{-\alpha}\left(q^{t+1}\right) F^{p}\left(q^{t}\right) \\
& \leq\left(\frac{p+\beta}{1-\alpha+\theta}\right)^{p} \sum_{t=\log _{q} a}^{\infty} \frac{k\left(q^{t}\right) v\left(q^{t}\right) w\left(q^{t+1}\right) r^{p}\left(q^{t}\right) f^{p}\left(q^{t}\right) G^{p-\alpha}\left(q^{t+1}\right)}{g^{p-1}\left(q^{t}\right)}
\end{aligned}
$$

where

$$
G(t)=(q-1) \sum_{s=\log _{q} a}^{\left(\log _{q} t\right)-1} q^{s} g\left(q^{s}\right) \text { and } \quad F(t)=(q-1) \sum_{s=\log _{q} t}^{\infty} q^{s} r\left(q^{s}\right) f\left(q^{s}\right)
$$

Theorem 3.21 Let $\mathbb{T}$ be a time scale with $a \in[0, \infty)_{\mathbb{T}}$. In addition, suppose $f, g, k, r, w, v$ are nonnegative $r d$-continuous functions on $[a, \infty)_{\mathbb{T}}$ such that $k$ is nonincreasing. Further, 
assume there exist $\theta, \beta \geq 0$ such that $\frac{w^{\Delta}(t)}{w(t)} \leq \theta\left(\frac{H^{\Delta}(t)}{H^{\sigma}(t)}\right)$ and $\frac{v^{\Delta}(t)}{v^{\sigma}(t)} \leq \beta\left(\frac{K^{\Delta}(t)}{K(t)}\right)$, where

$$
H(t)=\int_{t}^{\infty} g(s) \Delta s \quad \text { and } \quad K(t)=\int_{a}^{t} r(s) f(s) \Delta s, \quad t \in[a, \infty)_{\mathbb{T}} .
$$

If $p \geq 1,0 \leq \alpha<1$, then

$$
\begin{aligned}
& \int_{a}^{\infty} k^{\sigma}(t) v^{\sigma}(t) w(t) g(t) H^{-\alpha}(t)\left(K^{\sigma}(t)\right)^{p} \Delta t \\
& \quad \leq\left(\frac{p+\beta}{1-\alpha+\theta}\right)^{p} \int_{a}^{\infty} \frac{k^{\sigma}(t) v^{\sigma}(t) w(t) r^{p}(t) f^{p}(t) H^{p-\alpha}(t)}{g^{p-1}(t)} \Delta t .
\end{aligned}
$$

Proof Utilizing integration by parts formula on time scales (2.2) with

$$
u^{\Delta}(t)=w(t) g(t) H^{-\alpha}(t) \quad \text { and } \quad z^{\sigma}(t)=k^{\sigma}(t) v^{\sigma}(t)\left(K^{\sigma}(t)\right)^{p},
$$

we have

$$
\begin{aligned}
& \int_{a}^{\infty} k^{\sigma}(t) v^{\sigma}(t) w(t) g(t) H^{-\alpha}(t)\left(K^{\sigma}(t)\right)^{p} \Delta t \\
& \quad=\left[u(t) k(t) v(t) K^{p}(t)\right]_{a}^{\infty}+\int_{a}^{\infty}(-u(t))\left(k(t) v(t) K^{p}(t)\right)^{\Delta} \Delta t
\end{aligned}
$$

where

$$
u(t)=-\int_{t}^{\infty} w(s) g(s) H^{-\alpha}(s) \Delta s .
$$

From the chain rule on time scales (2.7), the product rule (2.1), and the assumption $\frac{w^{\Delta}(t)}{w(t)} \leq$ $\theta\left(\frac{H^{\Delta}(t)}{H^{\sigma}(t)}\right)$, we have

$$
\begin{aligned}
\left(-w(s) H^{1-\alpha}(s)\right)^{\Delta} & =-\left(w^{\Delta}(s)\left(H^{\sigma}(s)\right)^{1-\alpha}+w(s)\left(H^{1-\alpha}(s)\right)^{\Delta}\right) \\
& \geq-\left(\theta w(s)\left(H^{\sigma}(s)\right)^{-\alpha} H^{\Delta}(s)+(1-\alpha) w(s) H^{-\alpha}(c) H^{\Delta}(s)\right) .
\end{aligned}
$$

Because $H^{\Delta}(s)=-g(s) \leq 0, c \geq s$, and $0 \leq \alpha<1$, we get

$$
\begin{aligned}
\left(-w(s) H^{1-\alpha}(s)\right)^{\Delta} & \geq \theta w(s) g(s) H^{-\alpha}(s)+(1-\alpha) w(s) g(s) H^{-\alpha}(s) \\
& =(1-\alpha+\theta) w(s) g(s) H^{-\alpha}(s) .
\end{aligned}
$$

Thus

$$
w(s) g(s) H^{-\alpha}(s) \leq \frac{1}{1-\alpha+\theta}\left(-w(s) H^{1-\alpha}(s)\right)^{\Delta} .
$$

Hence

$$
\begin{aligned}
-u(t) & =\int_{t}^{\infty} w(s) g(s) H^{-\alpha}(s) \Delta s \leq \frac{1}{1-\alpha+\theta} \int_{t}^{\infty}\left(-w(s) H^{1-\alpha}(s)\right)^{\Delta} \Delta s \\
& =\frac{1}{1-\alpha+\theta} w(t) H^{1-\alpha}(t) .
\end{aligned}
$$


Applying the product rule (2.1) and the chain rule on time scales (2.7), we see that there exists $c \in[t, \sigma(t)]$ such that

$$
\begin{aligned}
\left(k(t) v(t) K^{p}(t)\right)^{\Delta} & =(k(t) v(t))^{\Delta} K^{p}(t)+k^{\sigma}(t) v^{\sigma}(t)\left(K^{p}(t)\right)^{\Delta} \\
& =k^{\Delta}(t) v(t) K^{p}(t)+k^{\sigma}(t) v^{\Delta}(t) K^{p}(t)+p k^{\sigma}(t) v^{\sigma}(t) K^{p-1}(c) K^{\Delta}(t) .
\end{aligned}
$$

Considering $k^{\Delta}(t) \leq 0, K^{\Delta}(t)=r(t) f(t) \geq 0, c \leq \sigma(t), p \geq 1$, and $\frac{v^{\Delta}(t)}{v^{\sigma}(t)} \leq \beta\left(\frac{K^{\Delta}(t)}{K(t)}\right)$, we get

$$
\begin{aligned}
\left(k(t) v(t) K^{p}(t)\right)^{\Delta} & \leq \beta k^{\sigma}(t) v^{\sigma}(t) r(t) f(t) K^{p-1}(t)+p k^{\sigma}(t) v^{\sigma}(t) r(t) f(t)\left(K^{\sigma}(t)\right)^{p-1} \\
& \leq(p+\beta) k^{\sigma}(t) v^{\sigma}(t) r(t) f(t)\left(K^{\sigma}(t)\right)^{p-1} .
\end{aligned}
$$

Combining (3.19), (3.20), and (3.21), we obtain (note that $K(a)=0$ and $u(\infty)=0$ )

$$
\begin{aligned}
& \int_{a}^{\infty} k^{\sigma}(t) v^{\sigma}(t) w(t) g(t) H^{-\alpha}(t)\left(K^{\sigma}(t)\right)^{p} \Delta t \\
& \quad \leq \frac{p+\beta}{1-\alpha+\theta} \int_{a}^{\infty} k^{\sigma}(t) v^{\sigma}(t) w(t) r(t) f(t) H^{1-\alpha}(t)\left(K^{\sigma}(t)\right)^{p-1} \Delta t .
\end{aligned}
$$

The last inequality can be rewritten as

$$
\begin{aligned}
\int_{a}^{\infty} & k^{\sigma}(t) v^{\sigma}(t) w(t) g(t) H^{-\alpha}(t)\left(K^{\sigma}(t)\right)^{p} \Delta t \\
\leq & \frac{p+\beta}{1-\alpha+\theta} \int_{a}^{\infty}\left(\left(k^{\sigma}(t) v^{\sigma}(t) w(t) g(t)\right)^{(p-1) / p} H^{-\alpha(p-1) / p}(t)\left(K^{\sigma}(t)\right)^{p-1}\right) \\
& \times\left(\frac{\left(k^{\sigma}(t) v^{\sigma}(t) w(t)\right)^{1 / p} r(t) f(t) H^{(p-\alpha) / p}(t)}{g^{(p-1) / p}(t)}\right) \Delta t .
\end{aligned}
$$

Using the dynamic Hölder inequality (2.8) with indices $p$ and $p /(p-1)$, we obtain

$$
\begin{aligned}
& \int_{a}^{\infty} k^{\sigma}(t) v^{\sigma}(t) w(t) g(t) H^{-\alpha}(t)\left(K^{\sigma}(t)\right)^{p} \Delta t \\
& \leq \frac{p+\beta}{1-\alpha+\theta}\left(\int_{a}^{\infty} k^{\sigma}(t) v^{\sigma}(t) w(t) g(t) H^{-\alpha}(t)\left(K^{\sigma}(t)\right)^{p} \Delta t\right)^{(p-1) / p} \\
& \quad \times\left(\int_{a}^{\infty} \frac{k^{\sigma}(t) v^{\sigma}(t) w(t) r^{p}(t) f^{p}(t) H^{p-\alpha}(t)}{g^{p-1}(t)} \Delta t\right)^{1 / p} .
\end{aligned}
$$

This implies that

$$
\begin{aligned}
& \int_{a}^{\infty} k^{\sigma}(t) v^{\sigma}(t) w(t) g(t) H^{-\alpha}(t)\left(K^{\sigma}(t)\right)^{p} \Delta t \\
& \quad \leq\left(\frac{p+\beta}{1-\alpha+\theta}\right)^{p} \int_{a}^{\infty} \frac{k^{\sigma}(t) v^{\sigma}(t) w(t) r^{p}(t) f^{p}(t) H^{p-\alpha}(t)}{g^{p-1}(t)} \Delta t .
\end{aligned}
$$

This concludes the proof.

Remark 3.22 In Theorem 3.21, if we set $k(t)=v(t)=w(t)=1$ and $r(t)=g(t)$, then we may take $\theta=\beta=0$, and in this case inequality (3.18) reduces to inequality (1.17). 
Corollary 3.23 If $\mathbb{T}=\mathbb{R}$ in Theorem 3.21, then, using relations (2.3), inequality (3.18) becomes

$$
\begin{aligned}
& \int_{a}^{\infty} k(t) v(t) w(t) g(t) H^{-\alpha}(t) K^{p}(t) d t \\
& \quad \leq\left(\frac{p+\beta}{1-\alpha+\theta}\right)^{p} \int_{a}^{\infty} \frac{k(t) v(t) w(t) r^{p}(t) f^{p}(t) H^{p-\alpha}(t)}{g^{p-1}(t)} d t
\end{aligned}
$$

where

$$
H(t)=\int_{t}^{\infty} g(s) d s \quad \text { and } \quad K(t)=\int_{a}^{t} r(s) f(s) d s
$$

Remark 3.24 In Corollary 3.23, if we set $k(t)=v(t)=w(t)=1, r(t)=g(t)$ and $a=0$, then we may take $\theta=\beta=0$, and in this case inequality (3.22) reduces to

$$
\int_{0}^{\infty} \frac{g(t)\left(\int_{0}^{t} g(s) f(s) d s\right)^{p}}{\left(\int_{t}^{\infty} g(s) d s\right)^{\alpha}} d t \leq\left(\frac{p}{1-\alpha}\right)^{p} \int_{0}^{\infty} g(t) f^{p}(t)\left(\int_{t}^{\infty} g(s) d s\right)^{p-\alpha} d t
$$

which is the continuous analogue of the discrete inequality (1.10).

Corollary 3.25 If $\mathbb{T}=h \mathbb{Z}$ in Theorem 3.21 , then, using relations (2.5), inequality (3.18) becomes

$$
\begin{aligned}
& \sum_{t=\frac{a}{h}}^{\infty} k(h t+h) v(h t+h) w(h t) g(h t) H^{-\alpha}(h t) K^{p}(h t+h) \\
& \quad \leq\left(\frac{p+\beta}{1-\alpha+\theta}\right)^{p} \sum_{t=\frac{a}{h}}^{\infty} \frac{k(h t+h) v(h t+h) w(h t) r^{p}(h t) f^{p}(h t) H^{p-\alpha}(h t)}{g^{p-1}(h t)}
\end{aligned}
$$

where

$$
H(t)=h \sum_{s=\frac{t}{h}}^{\infty} g(h s) \quad \text { and } \quad K(t)=h \sum_{s=\frac{a}{h}}^{\frac{t}{h}-1} r(h s) f(h s) .
$$

Corollary 3.26 For $\mathbb{T}=\mathbb{Z}$, we simply take $h=1$ in Corollary 3.25. In this case, inequality (3.18) becomes

$$
\begin{aligned}
& \sum_{t=a}^{\infty} k(t+1) v(t+1) w(t) g(t) H^{-\alpha}(t) K^{p}(t+1) \\
& \quad \leq\left(\frac{p+\beta}{1-\alpha+\theta}\right)^{p} \sum_{t=a}^{\infty} \frac{k(t+1) v(t+1) w(t) r^{p}(t) f^{p}(t) H^{p-\alpha}(t)}{g^{p-1}(t)}
\end{aligned}
$$

where

$$
H(t)=\sum_{s=t}^{\infty} g(s) \quad \text { and } \quad K(t)=\sum_{s=a}^{t-1} r(s) f(s) .
$$


Remark 3.27 In Corollary 3.26, if we set $k(t)=v(t)=w(t)=1, r(t)=g(t)$ and $a=1$, then we may take $\theta=\beta=0$, and in this case inequality (3.25) reduces to inequality (1.10).

Corollary 3.28 If $\mathbb{T}=\overline{q^{\mathbb{Z}}}$ in Theorem 3.21, then, using relations (2.6), inequality (3.18) becomes

$$
\begin{aligned}
& \sum_{t=\log _{q} a}^{\infty} k\left(q^{t+1}\right) v\left(q^{t+1}\right) w\left(q^{t}\right) g\left(q^{t}\right) H^{-\alpha}\left(q^{t}\right) K^{p}\left(q^{t+1}\right) \\
& \leq\left(\frac{p+\beta}{1-\alpha+\theta}\right)^{p} \sum_{t=\log _{q} a}^{\infty} \frac{k\left(q^{t+1}\right) v\left(q^{t+1}\right) w\left(q^{t}\right) r^{p}\left(q^{t}\right) f^{p}\left(q^{t}\right) H^{p-\alpha}\left(q^{t}\right)}{g^{p-1}\left(q^{t}\right)}
\end{aligned}
$$

where

$$
H(t)=(q-1) \sum_{s=\log _{q} t}^{\infty} q^{s} g\left(q^{s}\right) \quad \text { and } \quad K(t)=(q-1) \sum_{s=\log _{q} a}^{\left(\log _{q} t\right)-1} q^{s} r\left(q^{s}\right) f\left(q^{s}\right)
$$

Theorem 3.29 Assume $\mathbb{T}$ is a time scale with $a \in[0, \infty)_{\mathbb{T}}$. In addition, let $f, g, k, r, w, v$ be nonnegative rd-continuous functions on $[a, \infty)_{\mathbb{T}}$ such that $k$ is nondecreasing. Moreover, suppose there exist $\theta, \beta \geq 0$ such that $\frac{w^{\Delta}(t)}{w^{\sigma}(t)} \geq \theta\left(\frac{H^{\Delta}(t)}{H(t)}\right)$ and $\frac{v^{\Delta}(t)}{v(t)} \geq \beta\left(\frac{F^{\Delta}(t)}{F^{\sigma}(t)}\right)$, where

$$
H(t)=\int_{t}^{\infty} g(s) \Delta s \quad \text { and } \quad F(t)=\int_{t}^{\infty} r(s) f(s) \Delta s, \quad t \in[a, \infty)_{\mathbb{T}} .
$$

If $p \geq 1$ and $\alpha>\theta+1$, then

$$
\begin{aligned}
& \int_{a}^{\infty} k(t) v(t) w^{\sigma}(t) g(t) H^{-\alpha}(t) F^{p}(t) \Delta t \\
& \quad \leq\left(\frac{p+\beta}{\alpha-\theta-1}\right)^{p} \int_{a}^{\infty} \frac{k(t) v(t) w^{\sigma}(t) r^{p}(t) f^{p}(t) H^{\alpha(p-1)}(t)}{g^{p-1}(t)\left(H^{\sigma}(t)\right)^{p(\alpha-1)}} \Delta t .
\end{aligned}
$$

Proof From integration by parts formula on time scales (2.2), we have

$$
\begin{aligned}
& \int_{a}^{\infty} k(t) v(t) w^{\sigma}(t) g(t) H^{-\alpha}(t) F^{p}(t) \Delta t \\
& \quad=\left[u(t) k(t) v(t) F^{p}(t)\right]_{a}^{\infty}+\int_{a}^{\infty} u^{\sigma}(t)\left(-k(t) v(t) F^{p}(t)\right)^{\Delta} \Delta t,
\end{aligned}
$$

where

$$
u(t)=\int_{a}^{t} w^{\sigma}(s) g(s) H^{-\alpha}(s) \Delta s
$$

Using the chain rule on time scales (2.7), the product rule (2.1), and the hypothesis $\frac{w^{\Delta}(t)}{w^{\sigma}(t)} \geq$ $\theta\left(\frac{H^{\Delta}(t)}{H(t)}\right)$, we obtain

$$
\begin{aligned}
\left(w(s) H^{1-\alpha}(s)\right)^{\Delta} & =w^{\Delta}(s) H^{1-\alpha}(s)+w^{\sigma}(s)\left(H^{1-\alpha}(s)\right)^{\Delta} \\
& \geq \theta w^{\sigma}(s) H^{\Delta}(s) H^{-\alpha}(s)+(1-\alpha) w^{\sigma}(s) H^{-\alpha}(c) H^{\Delta}(s) .
\end{aligned}
$$


Since $H^{\Delta}(s)=-g(s) \leq 0, c \geq s$ and $\alpha>1$, we get

$$
\begin{aligned}
\left(w(s) H^{1-\alpha}(s)\right)^{\Delta} & \geq-\theta w^{\sigma}(s) g(s) H^{-\alpha}(s)+(\alpha-1) w^{\sigma}(s) g(s) H^{-\alpha}(s) \\
& =(\alpha-\theta-1) w^{\sigma}(s) g(s) H^{-\alpha}(s) .
\end{aligned}
$$

This gives us that

$$
w^{\sigma}(s) g(s) H^{-\alpha}(s) \leq \frac{1}{\alpha-\theta-1}\left(w(s) H^{1-\alpha}(s)\right)^{\Delta} .
$$

Therefore

$$
\begin{aligned}
u^{\sigma}(t) & =\int_{a}^{\sigma(t)} w^{\sigma}(s) g(s) H^{-\alpha}(s) \Delta s \leq \frac{1}{\alpha-\theta-1} \int_{a}^{\sigma(t)}\left(w(s) H^{1-\alpha}(s)\right)^{\Delta} \Delta s \\
& =\frac{1}{\alpha-\theta-1}\left(w^{\sigma}(t)\left(H^{\sigma}(t)\right)^{1-\alpha}-w(a) H^{1-\alpha}(a)\right) \\
& \leq \frac{1}{\alpha-\theta-1} w^{\sigma}(t)\left(H^{\sigma}(t)\right)^{1-\alpha} .
\end{aligned}
$$

Employing the product rule (2.1) and the chain rule on time scales (2.7), we see that there exists $c \in[t, \sigma(t)]$ such that

$$
\begin{aligned}
\left(-k(t) v(t) F^{p}(t)\right)^{\Delta} & =-\left((k(t) v(t))^{\Delta}\left(F^{\sigma}(t)\right)^{p}+k(t) v(t)\left(F^{p}(t)\right)^{\Delta}\right) \\
& =-\left(k^{\Delta}(t) v^{\sigma}(t)\left(F^{\sigma}(t)\right)^{p}+k(t) v^{\Delta}(t)\left(F^{\sigma}(t)\right)^{p}+p k(t) v(t) F^{p-1}(c) F^{\Delta}(t)\right) .
\end{aligned}
$$

As $k^{\Delta}(t) \geq 0, F^{\Delta}(t)=-r(t) f(t) \leq 0, c \geq t, p \geq 1$ and $\frac{v^{\Delta}(t)}{v(t)} \geq \beta\left(\frac{F^{\Delta}(t)}{F^{\sigma}(t)}\right)$, we have

$$
\begin{aligned}
\left(-k(t) v(t) F^{p}(t)\right)^{\Delta} & \leq \beta k(t) v(t) r(t) f(t)\left(F^{\sigma}(t)\right)^{p-1}+p k(t) v(t) r(t) f(t) F^{p-1}(t) \\
& \leq(p+\beta) k(t) v(t) r(t) f(t) F^{p-1}(t) .
\end{aligned}
$$

Combining (3.28), (3.29), and (3.30), we get (note that $F(\infty)=0$ and $u(a)=0)$

$$
\begin{aligned}
& \int_{a}^{\infty} k(t) v(t) w^{\sigma}(t) g(t) H^{-\alpha}(t) F^{p}(t) \Delta t \\
& \quad \leq \frac{p+\beta}{\alpha-\theta-1} \int_{a}^{\infty} k(t) v(t) w^{\sigma}(t) r(t) f(t)\left(H^{\sigma}(t)\right)^{1-\alpha} F^{p-1}(t) \Delta t,
\end{aligned}
$$

or equivalently,

$$
\begin{aligned}
\int_{a}^{\infty} & k(t) v(t) w^{\sigma}(t) g(t) H^{-\alpha}(t) F^{p}(t) \Delta t \\
\leq & \frac{p+\beta}{\alpha-\theta-1} \int_{a}^{\infty}\left(\left(k(t) v(t) w^{\sigma}(t) g(t)\right)^{(p-1) / p} H^{-\alpha(p-1) / p}(t) F^{p-1}(t)\right) \\
& \times\left(\frac{\left(k(t) v(t) w^{\sigma}(t)\right)^{1 / p} r(t) f(t) H^{\alpha(p-1) / p}(t)}{g^{(p-1) / p}(t)\left(H^{\sigma}(t)\right)^{\alpha-1}}\right) \Delta t .
\end{aligned}
$$


Applying the dynamic Hölder inequality (2.8) with indices $p$ and $p /(p-1)$, we get

$$
\begin{aligned}
& \int_{a}^{\infty} k(t) v(t) w^{\sigma}(t) g(t) H^{-\alpha}(t) F^{p}(t) \Delta t \\
& \leq \frac{p+\beta}{\alpha-\theta-1}\left(\int_{a}^{\infty} k(t) v(t) w^{\sigma}(t) g(t) H^{-\alpha}(t) F^{p}(t) \Delta t\right)^{(p-1) / p} \\
& \quad \times\left(\int_{a}^{\infty} \frac{k(t) v(t) w^{\sigma}(t) r^{p}(t) f^{p}(t) H^{\alpha(p-1)}(t)}{g^{p-1}(t)\left(H^{\sigma}(t)\right)^{p(\alpha-1)}} \Delta t\right)^{1 / p}
\end{aligned}
$$

which implies

$$
\begin{aligned}
& \int_{a}^{\infty} k(t) v(t) w^{\sigma}(t) g(t) H^{-\alpha}(t) F^{p}(t) \Delta t \\
& \quad \leq\left(\frac{p+\beta}{\alpha-\theta-1}\right)^{p} \int_{a}^{\infty} \frac{k(t) v(t) w^{\sigma}(t) r^{p}(t) f^{p}(t) H^{\alpha(p-1)}(t)}{g^{p-1}(t)\left(H^{\sigma}(f t)\right)^{p(\alpha-1)}} \Delta t,
\end{aligned}
$$

which is our desired result.

Remark 3.30 In Theorem 3.29, if we set $k(t)=v(t)=w(t)=1$ and $r(t)=g(t)$, then we may take $\theta=\beta=0$, and in this case inequality (3.27) reduces to a new form of inequality (1.18).

Corollary 3.31 If $\mathbb{T}=\mathbb{R}$ in Theorem 3.29, then, using relations (2.3), inequality (3.27) becomes

$$
\begin{aligned}
& \int_{a}^{\infty} k(t) v(t) w(t) g(t) H^{-\alpha}(t) F^{p}(t) d t \\
& \quad \leq\left(\frac{p+\beta}{\alpha-\theta-1}\right)^{p} \int_{a}^{\infty} \frac{k(t) v(t) w(t) r^{p}(t) f^{p}(t) H^{p-\alpha}(t)}{g^{p-1}(t)} d t
\end{aligned}
$$

where

$$
H(t)=\int_{t}^{\infty} g(s) d s \quad \text { and } \quad F(t)=\int_{t}^{\infty} r(s) f(s) d s .
$$

Remark 3.32 In Corollary 3.31, if we set $k(t)=v(t)=w(t)=1, r(t)=g(t)$ and $a=0$, then we may take $\theta=\beta=0$, and in this case inequality (3.31) reduces to

$$
\int_{0}^{\infty} \frac{g(t)\left(\int_{t}^{\infty} g(s) f(s) d s\right)^{p}}{\left(\int_{t}^{\infty} g(s) d s\right)^{\alpha}} d t \leq\left(\frac{p}{\alpha-1}\right)^{p} \int_{0}^{\infty} g(t) f^{p}(t)\left(\int_{t}^{\infty} g(s) f(s) d s\right)^{p-\alpha} d t
$$

which is the continuous analogue of the discrete inequality (1.13).

Corollary 3.33 If $\mathbb{T}=h \mathbb{Z}$ in Theorem 3.29 , then, using relations (2.5), inequality (3.27) becomes

$$
\begin{aligned}
& \sum_{t=\frac{a}{h}}^{\infty} k(h t) v(h t) w(h t+h) g(h t) H^{-\alpha}(h t) F^{p}(h t) \\
& \quad \leq\left(\frac{p+\beta}{\alpha-\theta-1}\right)^{p} \sum_{t=\frac{a}{h}}^{\infty} \frac{k(h t) v(h t) w(h t+h) r^{p}(h t) f^{p}(h t) H^{\alpha(p-1)}(h t)}{g^{p-1}(t) H^{p(\alpha-1)}(h t+h)},
\end{aligned}
$$


where

$$
H(t)=h \sum_{s=\frac{t}{h}}^{\infty} g(h s) \quad \text { and } \quad F(t)=h \sum_{s=\frac{t}{h}}^{\infty} r(h s) f(h s) .
$$

Corollary 3.34 For $\mathbb{T}=\mathbb{Z}$, we simply take $h=1$ in Corollary 3.33. In this case, inequality (3.27) becomes

$$
\begin{aligned}
& \sum_{t=a}^{\infty} k(t) v(t) w(t+1) g(t) H^{-\alpha}(t) F^{p}(t) \\
& \quad \leq\left(\frac{p+\beta}{\alpha-\theta-1}\right)^{p} \sum_{t=a}^{\infty} \frac{k(t) v(t) w(t+1) r^{p}(t) f^{p}(t) H^{\alpha(p-1)}(t)}{g^{p-1}(t) H^{p(\alpha-1)}(t+1)},
\end{aligned}
$$

where

$$
H(t)=\sum_{s=t}^{\infty} g(s) \quad \text { and } \quad F(t)=\sum_{s=t}^{\infty} r(s) f(s)
$$

Remark 3.35 In Corollary 3.34, if we set $k(t)=v(t)=w(t)=1, r(t)=g(t)$ and $a=1$, then we may take $\theta=\beta=0$, and in this case inequality (3.34) reduces to a new form of inequality (1.13).

Corollary 3.36 If $\mathbb{T}=\overline{q^{\mathbb{Z}}}$ in Theorem 3.29 , then, using relations (2.6), inequality (3.27) becomes

$$
\begin{aligned}
& \sum_{t=\log _{q} a}^{\infty} k\left(q^{t}\right) v\left(q^{t}\right) w\left(q^{t+1}\right) g\left(q^{t}\right) H^{-\alpha}\left(q^{t}\right) F^{p}\left(q^{t}\right) \\
& \leq\left(\frac{p+\beta}{\alpha-\theta-1}\right)^{p} \sum_{t=\log _{q} a}^{\infty} \frac{k\left(q^{t}\right) v\left(q^{t}\right) w\left(q^{t+1}\right) r^{p}\left(q^{t}\right) f^{p}\left(q^{t}\right) H^{\alpha(p-1)}\left(q^{t}\right)}{g^{p-1}\left(q^{t}\right) H^{p(\alpha-1)}\left(q^{t+1}\right)}
\end{aligned}
$$

where

$$
H(t)=(q-1) \sum_{s=\log _{q} t}^{\infty} q^{s} g\left(q^{s}\right) \quad \text { and } \quad F(t)=(q-1) \sum_{s=\log _{q} t}^{\infty} q^{s} r\left(q^{s}\right) f\left(q^{s}\right)
$$

\section{Conclusions}

In this work, by making use of the time scales' version of Hölder inequality, integration by parts formula, and Keller's chain rule, we obtained some original dynamic Hardy-type inequalities. The proved inequalities generalize some dynamic inequalities known in the literature, see remarks and corollaries after each theorem of our main results. In order to illustrate the theorems for each type of inequality, we applied them to various time scales such as $\mathbb{R}, h \mathbb{Z}, \overline{q^{\mathbb{Z}}}$, and $\mathbb{Z}$ as a subcase of $h \mathbb{Z}$. Possible future work includes studying different generalizations and variants of the dynamic Hardy inequality using the results presented in this article. 


\section{Acknowledgements}

We are immensely thankful to the editor and anonymous referees for their valuable remarks, which helped improve the paper.

\section{Funding}

Not applicable.

Availability of data and materials

Not applicable.

\section{Competing interests}

The authors announce that there are not any competing interests.

\section{Authors' contributions}

All authors have read and finalized the manuscript with equal contribution. All authors read and approved the final manuscript.

\section{Author details}

${ }^{1}$ Department of Mathematics, Faculty of Science, Al-Azhar University, Nasr City, Cairo, 11884, Egypt. ${ }^{2}$ Department of Mathematics, Cankaya University, Ankara, 06530, Turkey. ${ }^{3}$ Institute of Space Science, Magurele-Bucharest, Romania.

${ }^{4}$ Department of Medical Research, China Medical University Hospital, China Medical University, Taichung, Taiwan.

\section{Publisher's Note}

Springer Nature remains neutral with regard to jurisdictional claims in published maps and institutional affiliations.

Received: 21 April 2020 Accepted: 5 August 2020 Published online: 27 August 2020

\section{References}

1. Agarwal, R., O'Regan, D., Saker, S.: Dynamic Inequalities on Time Scales. Springer, Cham (2014)

2. Agarwal, R.P., Bohner, M., Peterson, A.: Inequalities on time scales: a survey. Math. Inequal. Appl. 4(4), 535-557 (2001)

3. Andersen, K.F., Heinig, H.P.: Weighted norm inequalities for certain integral operators. SIAM J. Math. Anal. 14(4), 834-844 (1983)

4. Andersen, K.F., Muckenhoupt, B.: Weighted weak type Hardy inequalities with applications to Hilbert transforms and maximal functions. Stud. Math. 72(1), 9-26 (1982)

5. Bennett, G.: Some elementary inequalities. Q. J. Math. Oxf. Ser. (2) 38(152), 401-425 (1987)

6. Bennett, G.: Some elementary inequalities. II. Q. J. Math. Oxf. Ser. (2) 39(156), 385-400 (1988)

7. Bennett, G.: Some elementary inequalities. III. Q. J. Math. Oxf. Ser. (2) 42(166), 149-174 (1991)

8. Bohner, M., Erbe, L., Peterson, A.: Oscillation for nonlinear second order dynamic equations on a time scale. J. Math. Anal. Appl. 301(2), 491-507 (2005)

9. Bohner, M., Peterson, A.: Dynamic Equations on Time Scales: An Introduction with Applications. Birkhäuser Boston, Boston (2001)

10. Bohner, M., Peterson, A.: Advances in Dynamic Equations on Time Scales. Birkhäuser Boston, Boston (2003)

11. Copson, E.T.: Note on series of positive terms. J. Lond. Math. Soc. 3(1), 49-51 (1928)

12. Copson, E.T.: Some integral inequalities. Proc. R. Soc. Edinb., Sect. A 75(2), 157-164 (1976)

13. Hardy, G.H.: Note on a theorem of Hilbert. Math. Z. 6(3-4), 314-317 (1920)

14. Hardy, G.H.: Notes on some points in the integral calculus. LX. Messenger Math. 54, 150-156 (1925)

15. Hardy, G.H.: Notes on some points in the integral calculus. LXIT. Messenger Math. 57, 12-16 (1928)

16. Hardy, G.H., Littlewood, J.E., Pólya, G.: Inequalities, 2nd edn. Cambridge University Press, Cambridge (1952)

17. Heinig, H.P.: Weighted norm inequalities for certain integral operators. II. Proc. Am. Math. Soc. 95(3), 387-395 (1985)

18. Hilger, S.: Analysis on measure chains-a unified approach to continuous and discrete calculus. Results Math. $18(1-2), 18-56(1990)$

19. Kac, V., Cheung, P.: Quantum Calculus. Universitext. Springer, New York (2002)

20. Kufner, A., Maligranda, L., Persson, L.-E.: The Hardy Inequality. About Its History and Some Related Results. Vydavatelský Servis, Plzeň (2007)

21. Kufner, A., Persson, L.-E.: Weighted Inequalities of Hardy Type. World Scientific, River Edge (2003)

22. Leindler, L.: Generalization of inequalities of Hardy and Littlewood. Acta Sci. Math. 31, 279-285 (1970)

23. Li, W.N.: Some new dynamic inequalities on time scales. J. Math. Anal. Appl. 319(2), 802-814 (2006)

24. Li, W.N.: Some Pachpatte type inequalities on time scales. Comput. Math. Appl. 57(2), 275-282 (2009)

25. Littlewood, J.E., Hardy, G.H.: Elementary theorems concerning power series with positive coefficients and moment constants of positive functions. J. Reine Angew. Math. 157, 141-158 (1927)

26. Opic, B., Kufner, A.: Hardy-Type Inequalities. Pitman Research Notes in Mathematics Series, vol. 219. Longman, Harlow (1990)

27. Řehák, P.: Hardy inequality on time scales and its application to half-linear dynamic equations. J. Inequal. Appl. 5, 495-507 (2005)

28. Saker, S.H., O'Regan, D., Agarwal, R.: Generalized Hardy, Copson, Leindler and Bennett inequalities on time scales. Math. Nachr. 287(5-6), 686-698 (2014) 